\title{
Positron anisotropy studies with PAMELA experiment
}

\section{B. Panico*, D. Campana, G. Osteria}

INFN, Sezione di Napoli, I-80126 Naples, Italy

E-mail: beatrice.panicoena.infn.it

\section{G. C. Barbarino, V. Scotti}

University of Naples 'Federico II', Department of Physics, I-80126 Naples, Italy

INFN, Sezione di Napoli, I-80126 Naples, Italy

\section{O. Adriani, M. Bongi, P. Spillantini, N. Mori}

University of Florence, Department of Physics and Astronomy, I-50019 Sesto

Fiorentino, Florence, Italy

INFN, Sezione di Firenze, I-50019 Sesto Fiorentino, Florence, Italy

\section{P. Papini, E. Vannuccini, S. Bottai}

INFN, Sezione di Firenze, I-50019 Sesto Fiorentino, Florence, Italy

\section{G. A. Bazilevskaya, A. N. Kvashnin, Y. I. Stozhkov}

Lebedev Physical Institute, RU-119991, Moscow, Russia

\section{R. Bellotti, A. Monaco}

University of Bari, Department of Physics, I-70126 Bari, Italy

INFN, Sezione di Bari, I-70126 Bari, Italy

\section{A. Bruno}

University of Bari, Department of Physics, I-70126 Bari, Italy

\section{F. Cafagna}

INFN, Sezione di Bari, I-70126 Bari, Italy

M. Boezio, V. Bonvicini, E. Mocchiutti, A. Vacchi, G. Zampa, N. Zampa

INFN, Sezione di Trieste, I-34149 Trieste, Italy

\section{E. A. Bogomolov, S. Y. Krutkov, G. I. Vasilyev}

Ioffe Physical Technical Institute, RU-194021 St. Petersburg, Russia

\section{P. Carlson,M. Pearce}

KTH, Department of Physics, and the Oskar Klein Centre for Cosmoparticle Physics, AlbaNova University Centre, SE-10691 Stockholm, Sweden

\section{Casolino}

INFN, Sezione di Roma Tor Vergata, I-00133 Rome, Italy

RIKEN, Advanced Science Institute, Wako-shi, Saitama, Japan 


\title{
G. Castellini
}

IFAC, I-50019 Sesto Fiorentino, Florence, Italy

C. De Donato, C. De Santis, L. Marcelli, M. Mergé, F. Palma, P. Picozza, R. Sparvoli University of Rome Tor Vergata, Department of Physics, I-00133 Rome, Italy

INFN, Sezione di Roma Tor Vergata, I-00133 Rome, Italy

\section{N. De Simone}

INFN, Sezione di Roma Tor Vergata, I-00133 Rome, Italy

\section{Di Felice}

INFN, Sezione di Roma Tor Vergata, I-00133 Rome, Italy

Agenzia Spaziale Italiana (ASI) Science Data Center, Via del Politecnico snc I-00133 Rome, Italy

\section{Formato, R. Munini}

University of Trieste, Department of Physics, I-34147 Trieste, Italy

INFN, Sezione di Trieste, I-34149 Trieste, Italy

\section{A. M. Galper,A. V. Karelin,S. V. Koldashov,S. Koldobskiy,Y. T. Yurkin,V. Malakhov,A.} Leonov,A. G. Mayorov,V. V. Mikhailov,S. A. Voronov

National Research Nuclear University MEPhI, RU-115409 Moscow

\section{U. Giaccari}

INFN, Sezione di Napoli, I-80126 Naples, Italy

Now at Universidade Federal do Rio de Janeiro, Instituto de Fisica, Rio de Janeiro, RJ, Brazil

\section{Martucci}

University of Rome Tor Vergata, Department of Physics, I-00133 Rome, Italy

INFN, Laboratori Nazionali di Frascati, Via Enrico Fermi 40, I-00044 Frascati, Italy

\section{W. Menn, M. Simon}

Universitat Siegen, Department of Physics, D-57068 Siegen, Germany

\section{Ricci}

INFN, Laboratori Nazionali di Frascati, Via Enrico Fermi 40, I-00044 Frascati, Italy

\section{S. B. Ricciarini}

INFN, Sezione di Firenze, I-50019 Sesto Fiorentino, Florence, Italy

IFAC, I-50019 Sesto Fiorentino, Florence, Italy

\begin{abstract}
The PAMELA experiment is collecting data since 2006; its results indicate the presence of a large flux of positrons respect to electrons in the cosmic ray spectrum above $10 \mathrm{GeV}$. This excess might also be originated in objects such as pulsars and microquasars or through dark matter annihilation. Here the electrons and positrons events collected by PAMELA have been analized searching for anisotropies.
\end{abstract}

Frontier Research in Astrophysics,

26-31 May 2014

Mondello (Palermo), Italy

\footnotetext{
* Speaker.
} 


\section{Introduction}

Cosmic rays (CRs) are relativistic particles hitting the Earth from the outer space. They spread over a wide range of energies, from $10^{10} \mathrm{eV}$ to $10^{20} \mathrm{eV}$, and their spectrum is well described by a power law, excepting for the low energy region which is affected by a modulation due to the effect of the Sun activity.

Although cosmic rays have been discovered more than 100 years ago, there are a lot of open questions, about their origin and acceleration mechanism. As they diffuse inside the Galaxy over a very long time under the effect of magnetic fields, their arrival direction distribution is highly randomized [1]; since 80's has been clearly observed an energy-dependent anisotropy of galactic cosmic rays, both in the northern [2, 3] and in the southern hemisphere [4]. Cosmic ray electrons and positrons with energy greater than $10 \mathrm{GeV}$, contrary to hadrons, lose rapidly their energy, due to synchrotron radiation emission and inverse Compton collisions with low-energy photons of the interstellar radiation field. But, recently, PAMELA [10] and Fermi [11] have revealed a large flux of high energy positrons and electrons.

To explain data the presence of additional sources have been supposed. But two different areas of interest have been explored. In the first case, the additional sources are identified with known astrophysical sources, like pulsars and SNRs. In the second case the extra production is due to exotic processes, like Dark Matter annihilation or decay. Therefore the detection of an excess in cosmic ray positrons with energies high enough to minimize both the geomagnetic field and any heliospheric effects, might reveal the presence of such nearby sources. Measurements of the positron anisotropy can also be used to constrain the diffusion of cosmic ray electrons and positrons in the galaxy.

\subsection{The PAMELA experiment}

PAMELA is a Payload for AntiMatter Matter Exploration and Light-nuclei Astrophysics. It is mounted on a pressurized container on board of the Russian satellite Resurs DK1 and is launched on June 2006 from the Baikonur cosmodrome. It has a quasi-polar elliptical orbit, which goes from 350 and $600 \mathrm{~km}$ of altitude, with an inclination of $70^{\circ}$. The layout was optimized for precision study of light particles and antiparticles in primary cosmic rays on a range of energy between tens of $\mathrm{MeV}$ and hundred of $\mathrm{GeV}$.

A schematic view of PAMELA apparatus is showed in Fig.1; it is $\sim 1.3 \mathrm{~m}$ high with a mass of $\sim 470 \mathrm{~kg}$ and contains a time of flight system (ToF), a magnetic spectrometer, an anticoincidence system, an electromagnetic calorimeter, and a neutron detector. The ToF system provides the main experimental trigger and allows the identification of albedo particles and the proton-electron separation below $1 \mathrm{GeV} / \mathrm{c}$. It is composed by 6 layers of plastic scintillators arranged into 3 planes which information, combined with those from the magnetic spectrometer, permit the determination of particle velocities.

The spectrometer is composed by a permanent magnet equipped with six double-sided micro-strip silicon sensors, allowing the determination of the sign of the charge and the rigidity of particles up to $1 \mathrm{TeV} / \mathrm{c}$. Also ionization losses are measured into the silicon planes, obtaining the absolute charge of the particle.

There are two different anti-coincidence systems which allow the rejection of secondary particles 


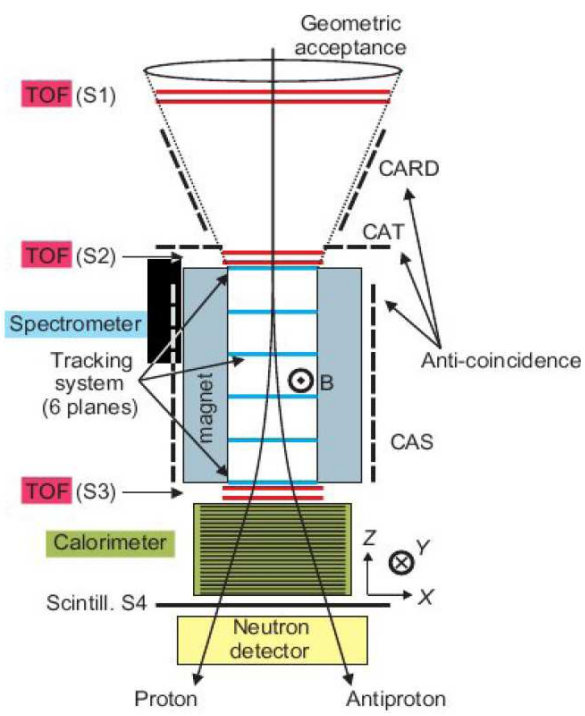

Figure 1: Schematic view of PAMELA experiment.

produced into the interaction with the mechanical structure of the experiment.

The electromagnetic calorimeter is composed by 44 silicon planes and 22 plates of tungsten. It provides a direct measurement of the energy for electrons and positrons and, through the analysis of the shower topology, allows the discrimination between hadrons and leptons, which is improved also by the neutron detector.

\section{The analysis}

For this analysis, the distribution of arrival direction of electrons and positrons with energy greater than $10 \mathrm{GeV}$ detected by PAMELA has been analized.

Due to the considered energies, cosmic ray electrons and positrons can be affected by the action of magnetic fields, causing an enhancement or a disappereance in the anisotropy signal. Therefore we correct the arrival direction of the particle for this effect. Starting from the reconstructed trajectory inside the instrument and the satellite position on the orbit, the particles were propagated back in the space up to about 20 radius of the Earth with the IGRF model. Also the albedo particles are rejected. The procedure above described is applied to PAMELA data which goes from July 2006 to January 2010, obtaining the positron and electron maps reported in Fig. 国. Maps are constructed using Healpix software and are represented in galactic coordinates whose equatorial plane is the projection of the plane of our Galaxy onto the celestial sphere.

The software HEALPix, which stands for Hierarchical Equal Area iso-Latitude Pixelization, allows a subdivision of a spherical surface in which each pixel covers the same surface area. The number of pixels is equal to $12 \cdot N_{\text {side }}^{2}$, where the parameter Nside which can only assume values equal to 2 or his multiples.

To obtain the background map, which corresponds to the hypothesis of an isotropic cosmic ray flux, we apply the shuffling method [5, 6] on the electron map; In this case, different data sets are 

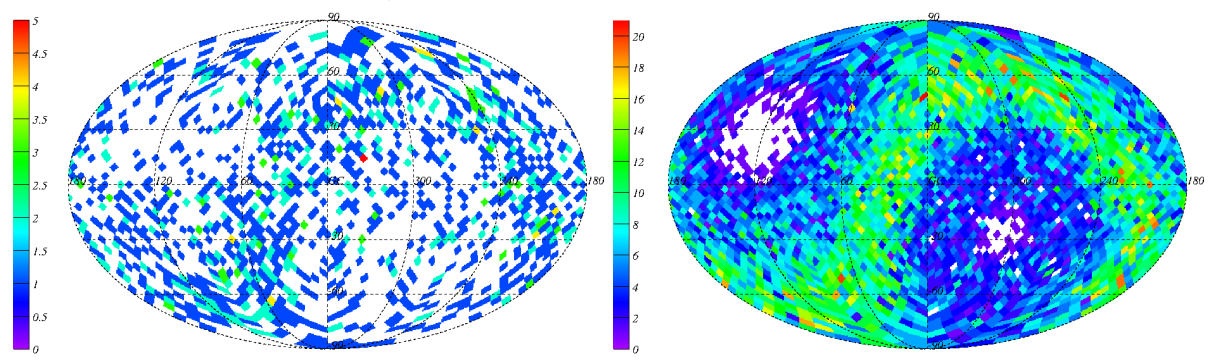

Figure 2: Electron and positron maps for $10 \mathrm{GeV}<\mathrm{E}<200 \mathrm{GeV}$, taking into account the geomagnetic field effect.

generated combining arrival direction of a chosen real event in the instrument local frame with the arrival time of another randomly selected real event. Therefore, the coverage map is the average of he different maps and is constructed starting from data itself and preserving the experimental exposure.

These maps are made up of uncorrelated bins, but to highlight an anisotropy signal with a definite angular scale, we need to have maps with bin size closer to that scale. Indeed, if the bin is too small, the anisotropy signal can be split among several adjacent bins, while if the bin is too large the background can conceal the signal. Therefore maps are integrated in order to have correlated bins over different integration radius, $10^{\circ}-30^{\circ}$.

The search for a significant excess or deficit of the signal over the background is calculated using the following formula suggested by Li and Ma in 1983 [8]

$$
S= \pm \sqrt{2}\left\{N_{\mathbf{o n}} \ln \left[\frac{1+\alpha}{\alpha}\left(\frac{N_{\mathbf{o n}}}{N_{\mathbf{o n}}+N_{\mathbf{o f f}}}\right)\right]+N_{\mathbf{o f f}} \ln \left[(1+\alpha)\left(\frac{N_{\mathbf{o f f}}}{N_{\mathbf{o n}}+N_{\mathbf{o f f}}}\right)\right]\right\}^{1 / 2}
$$

where:

- $N_{\text {on }}$ is the observed number of events in a given sky region $\mathrm{w}_{\text {on }}$ ('on' region);

- $N_{\text {off }}$ is the background number of events in the same sky region $\mathrm{w}_{\text {off }}$ ('off' region);

- $\alpha=\mathrm{w}_{\text {on }} / \mathrm{w}_{\text {off }}$, is the exposure weighted areas of the on and off source regions respectively, as in this case the on-source and off-source regions coincide so $\alpha=1$.

$N_{\mathbf{o n}}$ and $N_{\text {off }}$ are independent measurements and in the absence of any signal, the significance $S$, which represents the confidence level of the observational result, approximates a gaussian variable with mean equal to zero. Significance maps are constructed by comparing signal and background maps in pairs for the different integration radii and are reported in Fig.3.

\section{Discussion and conclusions}

The analysis on cosmic positron anisotropy based on PAMELA data has been described. More than $2 \cdot 10^{4}$ events in the energy range from $10 \mathrm{GeV}$ to $200 \mathrm{GeV}$ have been analized, taking into 

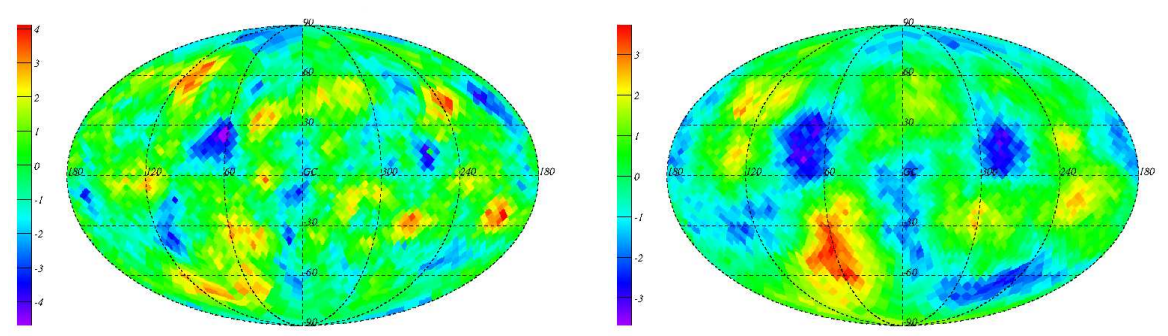

Figure 3: Significance maps for $10 \mathrm{GeV}<\mathrm{E}<200$, over the following angular scales: $10^{\circ}, 30^{\circ}$.

account also the effect of magnetic field. No excess in the arrival distribution of events has been highlighted; further improvements are ongoing, especially in the development of new procedure of analysis.

\section{References}

[1] Longair, M.S.: 1992, in High energy astrophysics, Cambridge University Press.

[2] Nagashima, K., Fujimoto, K., Jacklyn, R.M., 1998, J. of Geophys. Res, 17429.

[3] Aglietta, M., et al., 2009, Astrophys. J. Lett., 692, L130.

[4] Abbasi,R., et al., 2010, Astrophys. J. Lett., 718, L194.

[5] Cassiday, G., 1990, Nucl. Phys. B (Proc. Suppl.), 14, 291.

[6] Alexandreas, D.E., et al., 1991, Nucl. Inst. and Meth. A, 328, 570.

[7] Gorski, K.M., Hivon, E., Banday, A.J., Wandelt, B.D., Hansen, F.K., Reinecke, M., Bartelmann, M., 2005, Astrophys. Journ., 622, 759.

[8] Li, T.P., Ma, Y.Q., 1983, Astrophys. Journ., 272, 317.

[9] http://www.ngdc.noaa.gov/IAGA/vmod/igrf.html

[10] Adriani, O., et al., 2009, Nature, 458, 486.

[11] Ackermann, M., et al., 2012, Phys. Rev. Lett., 108, 011103.

\section{DISCUSSION}

JAMES H. BEALL: Can you remind us of the orbit of the satellite and inclination with respect to the Earth's equator?

BEATRICE PANICO: Yes, sure. PAMELA has a quasi polar and elliptical orbit with an inclination of $70^{\circ}$ and an altitude which goes from about 300 to $600 \mathrm{~km}$. 